Original

\title{
Effects of parathyroid hormone dosage and schedule on bone regeneration
}

\author{
Katsuyoshi Tsunori \\ Division of Applied Oral Sciences, Nihon University Graduate School of Dentistry, Tokyo, Japan \\ (Received December 14, 2014; Accepted April 6, 2015)
}

\begin{abstract}
This study investigated the effects of administration of parathyroid hormone (PTH) at different dosages and schedules on bone regeneration in critical-size bone defects in rat calvariae. After calvarial defects had been prepared in 50 rats, they were divided into five treatment groups: $15 \mu \mathrm{g} / \mathrm{kg}$ PTH daily (PTH-15), $35 \mu \mathrm{g} / \mathrm{kg}$ PTH three times per week (PTH-35), $105 \mu \mathrm{g} / \mathrm{kg}$ once per week (PTH-105-1), 105 $\mu \mathrm{g} / \mathrm{kg}$ three times per week (PTH-105-3), and controls given vehicle alone. Bone regeneration was evaluated radiographically using micro-computed tomography (micro-CT) or histologically. The amount of newly generated bone in the calvarial defects was found to be significantly greater in the PTH groups than in the control group, and did not differ significantly among the PTH-15, PTH-35, and PTH-105-1 groups, whereas the PTH-105-3 group showed a significantly greater degree of new bone formation than the other PTH groups. It appeared that a higher dose of PTH stimulated a greater degree of bone regeneration in this experimental setting. The present results also suggest that the total dose of PTH administered is significantly related to the amount of bone regenerated within a defined period, indicating that less frequent administration of PTH might be a feasible protocol for bone regeneration therapy.
\end{abstract}

(J Oral Sci 57, 131-136, 2015)

Correspondence to Dr. Katsuyoshi Tsunori, c/o Dr. Shuichi Sato, Department of Periodontology, Nihon University School of Dentistry, 1-8-13 Kanda-Surugadai, Chiyoda-ku, Tokyo 101-8310, Japan

Fax:+81-3-3219-8349 E-mail: satou.shuuichi@nihon-u.ac.jp

doi.org/10.2334/josnusd.57.131

DN/JST.JSTAGE/josnusd/57.131
Keywords: parathyroid hormone; administration protocol; bone regeneration therapy; rat calvaria; micro-CT.

\section{Introduction}

Various clinical protocols for regeneration of bone tissue have been introduced $(1,2)$. However, few of them have been effective for treatment of severe alveolar bone loss in patients with periodontal disease.

Parathyroid hormone (PTH) is a major regulator of bone remodeling and calcium homeostasis, exerting both anabolic and catabolic effects in bone tissue. Continuous treatment with PTH causes bone resorption, whereas intermittent PTH treatment promotes bone formation (3). Injection of rats with $\mathrm{PTH}$ accelerates the differentiation of osteoprogenitor cells into osteoblasts (4). Administration of PTH also inhibits osteoblast apoptosis, causing prolonged, enhanced bone formation (5). The anabolic effects of intermittent PTH administration are beneficial in patients with severe osteoporosis, increasing bone mass and reducing the incidence of fractures (6).

Recently, the possible utilization of PTH for regenerative applications, such as fracture healing and treatment of osseous defects, in the craniofacial region has attracted interest (7). However, little information on the reparative effects of PTH at various dosages for various periods on bone defects has been available. Therefore, the purpose of the present study was to evaluate the effects of various schedules and dosages of PTH on the regeneration of critical-size bone defects in rat calvaria.

\section{Materials and Methods}

Animals

Fifty 12-week-old male Fischer rats weighing 250-300 g were used. The animals were housed in metal cages in an 
animal room (temperature $22^{\circ} \mathrm{C}, 55 \%$ relative humidity, 12/12-h light/dark cycle) and fed a standard laboratory diet and water. This study was approved by the Animal Experimentation Committee of Nihon University School of Dentistry, Japan (AP13D018).

\section{PTH}

Synthetic human PTH (1-34 PTH; Asahi Kasei Pharma, Tokyo, Japan) was dissolved in $0.1 \mathrm{M}$ Tris- $\mathrm{HCl}$, at $\mathrm{pH}$ 7.5 , containing $2 \%$ bovine serum albumin.

\section{Surgical procedure}

The animals were premedicated by inhalation of isoflurane and then general anesthesia was induced by intraperitoneal injection of a mixture of $0.15 \mathrm{mg} / \mathrm{kg}$ dexmedetomidine hydrochloride, $2.0 \mathrm{mg} / \mathrm{kg}$ midazolam, and $2.5 \mathrm{mg} / \mathrm{kg}$ butorphanol tartrate. The forehead of each rat was also locally anesthetized with an injection of $0.5 \mathrm{~mL}$ of 1:8-diluted lidocaine (Xylocaine; Astra Zeneca, Osaka, Japan). The dorsal cranium was shaved and prepared for surgery aseptically. A horseshoe-shaped skin incision was made over the head, the parietal area was exposed under aseptic conditions, and the periosteum was elevated to expose the bone. Using a trephine burr constantly cooled with sterile saline, critical-size (5.0-mm diameter) bone defects were created on the right side of the parietal bone to avoid the sagittal suture (Fig. 1). The resulting calvarial disk was then removed carefully to avoid tearing the dura. After thoroughly rinsing the area with physiological saline to wash out any bone fragments, the skin was closed using 4-0 silk (Ethicon, Somerville, NJ, USA).

\section{PTH administration}

The treated rats were divided into five groups, which received PTH by subcutaneous injection at $15 \mu \mathrm{g} / \mathrm{kg}$ daily (PTH-15), $35 \mu \mathrm{g} / \mathrm{kg}$ PTH three times a week (PTH35), $105 \mu \mathrm{g} / \mathrm{kg}$ PTH once a week (PTH-105-1) or 105 $\mu \mathrm{g} / \mathrm{kg}$ PTH three times a week (PTH-105-3). The control animals were given only vehicle (sterile saline) each day during the experiment (Fig. 2). The first day of PTH administration and surgery was designated as day 0 .

\section{Measurement of serum calcium $\left(\mathrm{Ca}^{2+}\right)$ concentration and alkaline phosphatase (ALP) activity}

Under ether anesthesia, $\sim 80 \mu \mathrm{L}$ of blood was obtained from the subclavian vein every day for one week after surgery. The blood was centrifuged and the serum was stored at $-80^{\circ} \mathrm{C}$. Total serum $\mathrm{Ca}^{2+}$ and ALP were determined using a detection kit with automated clinical chemistry analyzers (7180 automated analyzer, Hitachi,

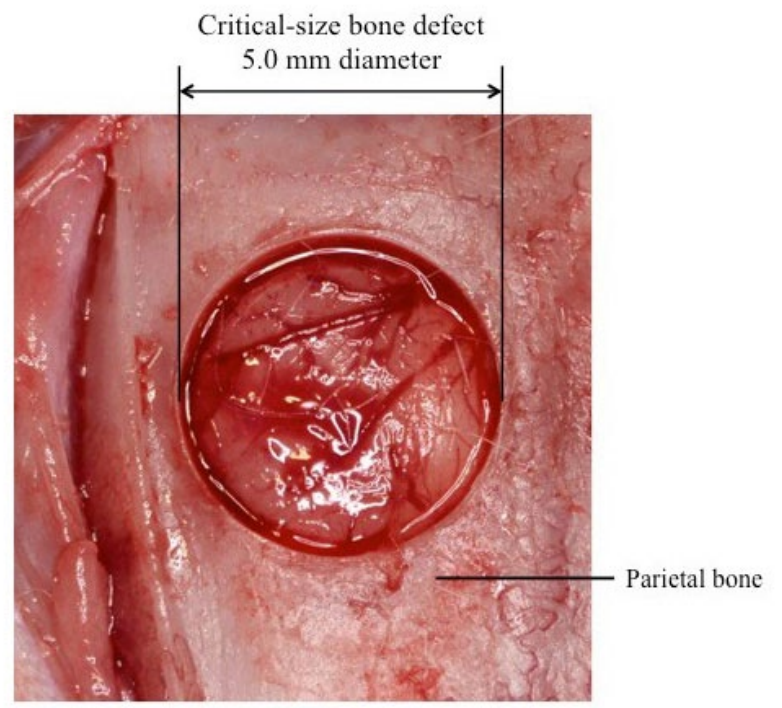

Fig. 1 Critical-size calvarial bone defects (5-mm diameter) were created in the dorsal part of the right parietal bone.

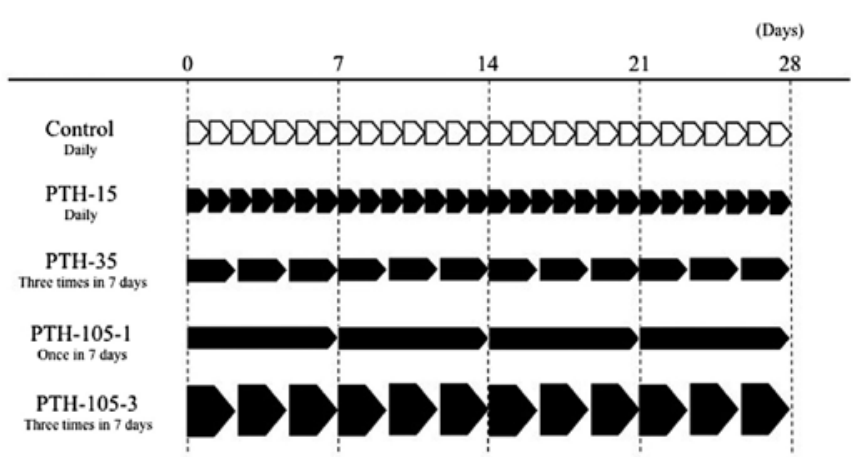

Fig. 2 Schedule of PTH administration.

Ibaraki, Japan).

Micro-computed tomography (micro-CT) analysis A micro-CT apparatus (R_mCT; Rigaku, Tokyo, Japan) was utilized to take images on days $0,7,14,21$, and 28 to examine bone regeneration in vivo. The images were reconstructed on a personal computer using the $i$-View software package (Rigaku, $i$-View Image Center, Tokyo, Japan). On day 0, a screen cylinder was drawn to define the initial bone defect. The bone volume (BV) was measured as the bone tissue within the cylinder on voxel images using BV measurement software (Kitasenjyu Radist Dental Clinic, $i$-View Image Center, Tokyo, Japan), which calculates the gray values and corresponding number of voxels in the regions of interest.

\section{Histological and histometric analyses}

The animals were sacrificed at 28 days after surgery. The skin was dissected, and the defect sites were removed, 


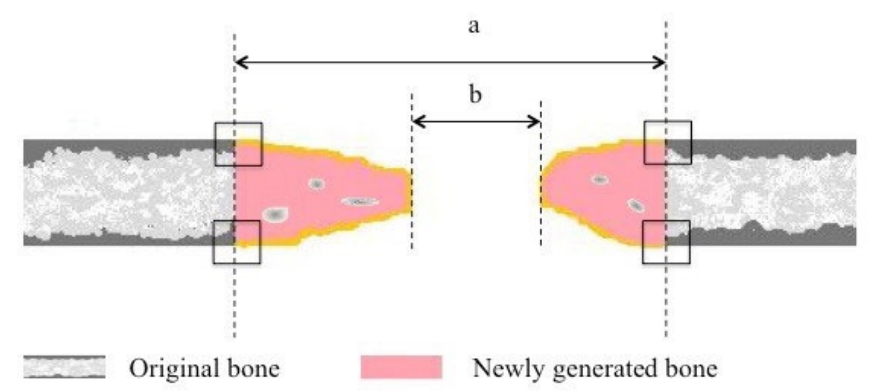

The area for counting osteoblast-like cells

Defect closure $(\%)=(a-b) / a \times 100$

Fig. 3 Schematic drawing of regenerative bone in calvarial defects and histometric parameters.

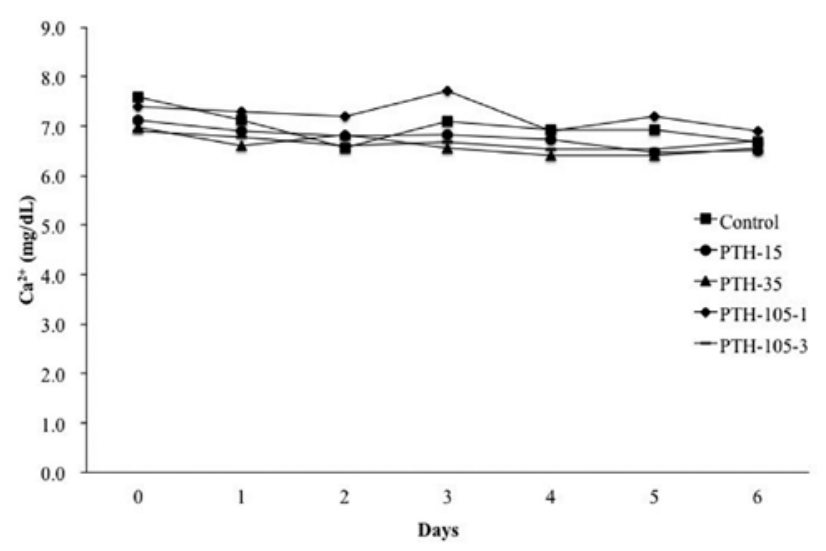

Fig. 4 Concentration of serum $\mathrm{Ca}^{2+}$.

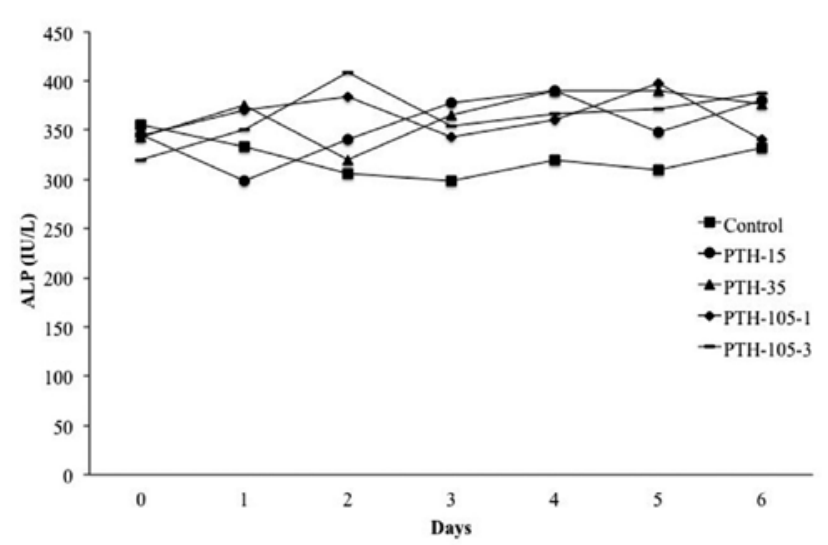

Fig. 5 Activity of serum ALP.

along with the surrounding bone and soft tissues. The specimens were then fixed in 10\% neutral buffered formalin, decalcified in 10\% EDTA for one week, and embedded in paraffin. Coronal sections ( $5 \mu \mathrm{m}$ thick) were prepared through the center of each circular defect, stained with hematoxylin and eosin, and examined under a light microscope equipped with a morphometric system
(Model BHF-142; Olympus, Tokyo, Japan).

Closure of each bone defect was determined by measuring the distance between the defect margins, and was expressed as a percentage of the width of the original bone defect. The osteoblast-like cells were counted in four areas at the edge of the original defects and expressed as averages (Fig. 3).

\section{Statistical analysis}

Means and standard deviations were calculated for the reossification ratio on days $7,14,21$, and 28 . The mean $\mathrm{BV}$, defect closure, and the numbers of osteoblasts-like cells were compared among the groups using the KruskalWallis rank test. The level of statistical significance was set at 0.05 . Statistical analyses were performed using the SPSS software package (Ver. 16.0J for Windows; SPSS, Inc., Chicago, IL, USA).

\section{Results \\ Concentration of serum $\mathrm{Ca}^{2+}$ and activity of serum ALP}

There were no significant differences in the serum $\mathrm{Ca}^{2+}$ levels among the PTH and control groups. The PTH groups tended to have higher serum ALP levels than the control group, but these differences were not significant (Figs. 4, 5).

\section{Micro-CT analysis}

In the micro-CT images, the radiopacity increased gradually in a time-dependent manner in all groups. The outline of the bone defect in the PTH groups was blurred and an irregular high-density region was observed in the defect area. In the PTH groups, there was significantly greater radiopacity at 21 and 28 days, compared with the control group (Fig. 6).

The BV was significantly higher in the PTH groups than in the control group at days 21 and 28 . The BV did not differ significantly among the PTH-15, PTH-35, and PTH-105-1 groups, but the PTH-105-3 group had significantly more new bone at day 28 (Fig. 7).

\section{Histological and histometric observations}

The PTH groups showed significant formation of new bone throughout the defects, relative to the control group. The PTH-105-3 group showed considerably thick lamellar bone. The control group showed limited bone formation with fibrous connective tissue (Fig. 8).

The closure of bone defects was enhanced significantly in the PTH groups relative to the control group. The PTH-105-3 group exhibited significantly greater defect closure than the other groups (Table 1). 


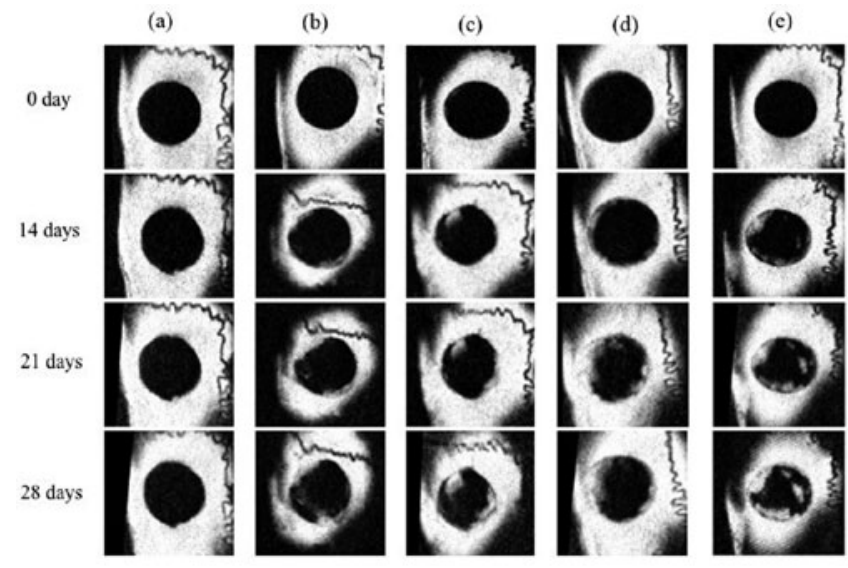

Fig. 6 Micro-CT images of critical-size bone defects at days 7-28: (a) Control, (b) PTH-15, (c) PTH-35, (d) PTH-105-1, and (e) PTH-105-3.

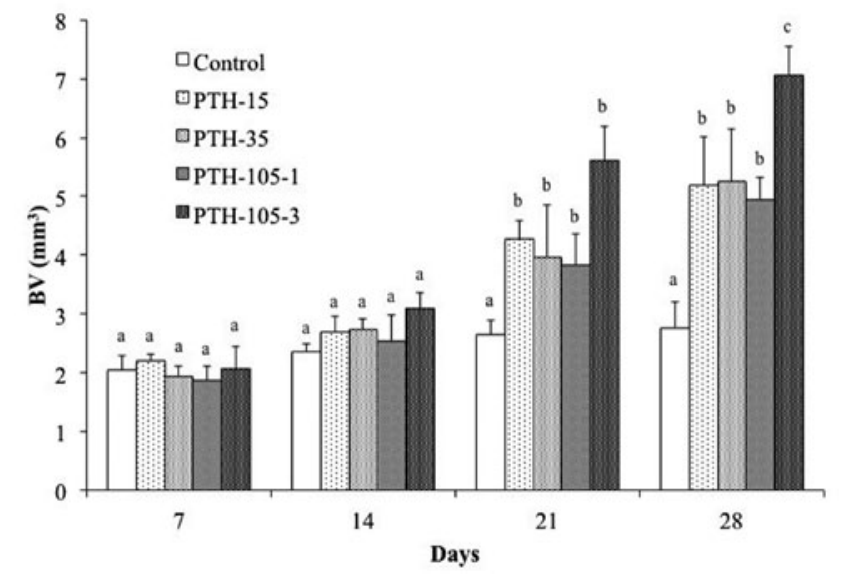

Fig. 7 Regenerative bone volume in critical-size bone defects. The same lower case letters on the same days indicate no statistical difference, Kruskal-Wallis rank test $(P>0.05)$.

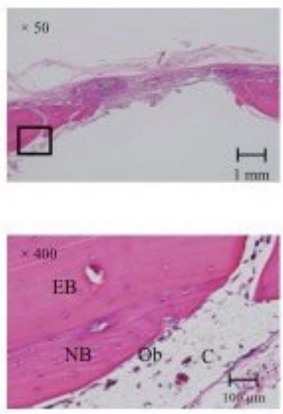

(a)
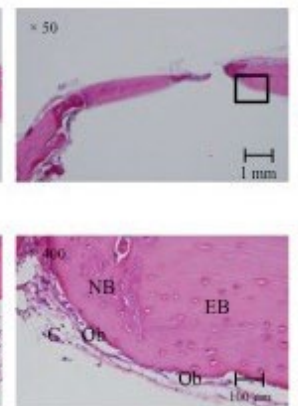

(b)
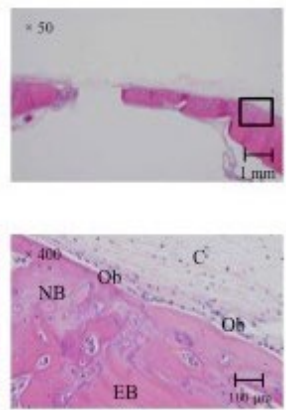

(c)
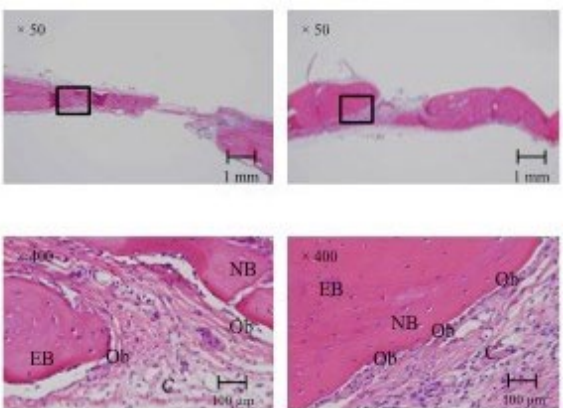

(d)

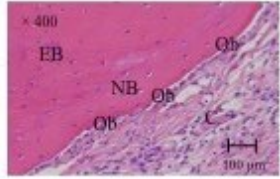

(e)

\section{NB: newly generated bone \\ EB: existing bone \\ $\mathrm{Ob}$ : osteoblast-like cells \\ $\mathrm{C}$ : connective tissue}

Fig. 8 Histological sections of critical-size bone defects at 28 days. The boxed area is the high-magnification histology. (a) Control, (b) PTH-15, (c) PTH-35, (d) PTH-105-1, and (e) PTH-105-3.

More osteoblast-like cells were observed at the edge of the original bone defect in the PTH groups. The greatest number of osteoblast-like cells was present in the PTH-105-3 group (Table 2).

\section{Discussion}

Intermittent administration of PTH has an anabolic effect, promoting the healing of bone defects. The results of this study indicated that the amount of newly generated bone in critical-size bone defects was significantly higher, and bone defect healing was faster, in the PTH groups than in the control group.

Various dosages of PTH have been used to promote regeneration of new bone. PTH administered at 60 $\mu \mathrm{g} / \mathrm{kg}$ was reported to increase bone deposition in rat parietal critical-size bone defects (8). Moreover, PTH at $25 \mu \mathrm{g} / \mathrm{kg}$ accelerated new bone formation in rabbit mandible critical-size bone defects (9). Yun et al. (10) and Stancoven et al. (11) showed that PTH administration at $15 \mu \mathrm{g} / \mathrm{kg}$ increased bone formation in rat calvarial bone defects. Accordingly, the lowest reported dose of $\mathrm{PTH}, 15 \mu \mathrm{g} / \mathrm{kg}$, was used in the present study. However, the optimal dose and schedule for enhancing bone defect healing remain unclear. In the present study, bone regeneration was enhanced significantly in the PTH-105-3 group relative to the other three groups, PTH-15, -35, and PTH-105-1, suggesting that a greater dose of PTH stimulated regeneration of more bone in rat critical-size bone defects. In contrast, there were no significant differences in bone regeneration among the PTH-15, -35 , and PTH-105-1 groups. Because the cumulative dosage of PTH administered to these three groups was the same, it 
Table 1 Percentage of bone closure rate

\begin{tabular}{ll}
\hline Group & Closure of bone defects $(\%)$ \\
\hline Control & $10.3 \pm 7.3^{\mathrm{a}}$ \\
PTH-15 & $40.3 \pm 15.2^{\mathrm{b}}$ \\
PTH-35 & $48.9 \pm 12.4^{\mathrm{b}}$ \\
PTH-105-1 & $55.3 \pm 4.2^{\mathrm{b}}$ \\
PTH-105-3 & $66.2 \pm 9.3^{\mathrm{c}}$ \\
\hline
\end{tabular}

The values with the same superscript letters indicate no statistical difference, group means $\pm \mathrm{SD}$, Kruskal-Wallis rank test $(P>0.05)$

could be considered that the total amount of PTH delivered during the experimental period is a significant factor for regenerative bone healing.

Daily intermittent subcutaneous administration of PTH has been reported to enhance bone regeneration $(12,13)$. However, as daily injection of PTH would be stressful to patients, an effective schedule for optimal PTH administration needs to be considered. The amount of bone regenerated in the PTH-15, -35, and PTH-105-1 groups was approximately the same, but the total frequency of PTH administration differed among the groups. In other words, a lower frequency of PTH administration using a larger dosage, and a higher frequency with a small dosage might be both equally effective for achieving a given degree of bone regeneration, as long as the total cumulative amount of PTH is the same. This concept would be useful when considering a better schedule of PTH administration.

PTH is a peptide hormone that regulates calcium and phosphate metabolism. Continuous infusion of PTH has been reported to induce catabolic effects in bones, resulting in hypercalcemia (14). In the present study, blood $\mathrm{Ca}^{2+}$ levels did not increase in the experimental groups relative to the control group, suggesting indicates that the dose and frequency of PTH administration in this study exerted anabolic effects. On the other hand, serum ALP levels showed a tendency to increase, suggesting active bone turnover, as the two are known to be correlated with each other (15).

Our histological observations showed that PTH stimulated new bone formation with an increase in the number of osteoblasts around the existing bone. A previous study had suggested that this increase in the number of osteoblasts was not dependent on the proliferation of osteoprogenitor cells or osteoblastogenesis; rather, PTH affected existing cells (16). These lining cells might undergo transition to an active form and resume matrix synthesis in response to PTH treatment.

In conclusion, the present findings suggest that the greater the dose of $\mathrm{PTH}$, the more bone is regenerated
Table 2 Number of osteoblast-like cells observed around the edge of the existing bone

\begin{tabular}{lc}
\hline Group & Number of cells \\
\hline Control & $70 \pm 8^{\mathrm{a}}$ \\
PTH-15 & $121 \pm 30^{\mathrm{b}}$ \\
PTH-35 & $146 \pm 22^{\mathrm{b}}$ \\
PTH-105-1 & $129 \pm 29^{\mathrm{b}}$ \\
PTH-105-3 & $244 \pm 35^{\mathrm{c}}$ \\
\hline
\end{tabular}

The values with the same superscript letters indicate no statistical difference, group means $\pm \mathrm{SD}$, Kruskal-Wallis rank test $(P>0.05)$

in critical-size rat calvarial bone defects, and that the total dose of PTH administered within a defined period is significant for bone regeneration. Therefore, a lower frequency of PTH administration with a higher dose could be a possible protocol design for bone regeneration therapy.

\section{Acknowledgments}

I would like to express my deepest gratitude to directors professor Masashi Miyazaki, professor Koichi Ito and associate professor Shuichi Sato for their support throughout the research. I would also like to express my deep appreciation to professor Yoshinori Arai for technical supervision of micro-CT analysis. I am grateful to Asahi Kasei Pharma (Tokyo, Japan) for providing the PTH.

\section{References}

1. Retzepi M, Donos N (2010) Guided bone regeneration: biological principle and therapeutic applications. Clin Oral Implants Res 21, 567-576.

2. Ramseier CA, Rasperini G, Batia S, Giannobile WV (2012) Advanced reconstructive technologies for periodontal tissue repair. Periodontol 2000 59, 185-202.

3. Mitlak BH (2002) Parathyroid hormone as a therapeutic agent. Curr Opin Pahrmacol 2, 694-699.

4. Skripitz R, Aspenberg P (2004) Parathyroid hormone--a drug for orthopedic surgery? Acta Orthop Scand 75, 654-662.

5. Jilka RL, Weinstein RS, Bellido T, Roberson P, Parfitt AM, Manolagas SC (1999) Increased bone formation by prevention of osteoblast apoptosis with parathyroid hormone. J Clin Invest 104, 439-446.

6. Neer RM, Arnaud CD, Zanchetta JR, Prince R, Gaich GA, Reginster JY et al. (2001) Effect of parathyroid hormone (1-34) on fractures and bone mineral density in postmenopausal women with osteoporosis. N Engl J Med 344, 1434-1441.

7. Chan HL, McCauley LK (2013) Parathyroid hormone applications in the craniofacial skeleton. J Dent Res 92, 18-25.

8. Andreassen TT, Cacciafesta V (2004) Intermittent parathyroid hormone treatment enhances guided bone regeneration in rat calvarial bone defects. J Craniofac Surg 15, 424-427.

9. Tang ZL, Zhang WJ, Wang DX, Chen JM, Ma H, Wu DR (2014) An experimental study addressing the promotion of 
mandibular defect repair through the intermittent subcutaneous injection of parathyroid hormone. J Oral Maxillofac Surg 72, 419-430.

10. Yun JI, Wikesjö UM, Borke JL, Bisch FC, Lewis JE, Herold RW et al. (2010) Effect of systemic parathyroid hormone (1-34) and a beta-tricalcium phosphate biomaterial on local bone formation in a critical-size rat calvarial defect model. J Clin Periodontol 37, 419-426.

11. Stancoven BW, Lee J, Dixon DR, McPherson JC III, Bisch FC, Wikesjö UM et al. (2013) Effect of bone morphogenetic protein-2, demineralized bone matrix and systemic parathyroid hormone (1-34) on local bone formation in a rat calvaria critical-size defect model. J Periodont Res 48, 243-251.

12. Ejersted C, Andreassen TT, Oxlund H, Jørgensen PH, Bak B, Häggblad J et al. (1993) Human parathyroid hormone (1-34) and (1-84) increase the mechanical strength and thickness of cortical bone in rats. J Bone Miner Res 8, 1097-1101.

13. Dobnig H, Turner RT (1997) The effects of programmed administration of human parathyroid hormone fragment (1-34) on bone histomorphometry and serum chemistry in rats. Endocrinology 138, 4607-4612.

14. Iida-Klein A, Lu SS, Kapadia R, Burkhart M, Moreno A, Dempster DW et al. (2005) Short-term continuous infusion of human parathyroid hormone 1-34 fragment is catabolic with decreased trabecular connectivity density accompanied by hypercalcemia in C57BL/J6 mice. J Endocrinol 186, 549-557.

15. Nozaka K, Miyakoshi N, Kasukawa Y, Maekawa S, Noguchi H, Shimada Y (2008) Intermittent administration of human parathyroid hormone enhances bone formation and union at the site of cancellous bone osteotomy in normal and ovariectomized rats. Bone 42, 90-97.

16. Dobnig H, Turner RT (1995) Evidence that intermittent treatment with parathyroid hormone increases bone formation in adult rats by activation of bone lining cells. Endocrinology $136,3632-3638$. 